\title{
Développement d'un cours francophone en ligne sur les politiques publiques en santé : une collaboration internationale
}

\section{Development of a French-language online health policy course: an international collaboration}

\author{
Réjean Hébert ${ }^{1}$, Yves Coppieters ${ }^{2}$, Christian Pradier ${ }^{3}$, Bryn Williams-Jones ${ }^{4}$, Cora Brahimi ${ }^{5}$, Céline Farley ${ }^{6}$
}

\section{$\hookrightarrow$ Résumé}

Objectif : Présenter le processus et les défis du développement d'un cours en ligne sur les politiques publiques en santé, conçu en collaboration internationale selon une approche par compétences.

Méthodes : Cinq experts de santé publique épaulés d'un expert en technologie éducative ont travaillé à l'élaboration du cours en adoptant une démarche rigoureuse : analyse des besoins, élaboration des cibles et des compétences visées, développement d'un scénario pédagogique comportant pour chaque module la cible visée, les éléments de contenu, la méthode d'enseignement, les activités d'apprentissage, le matériel à identifier ou à développer et les responsabilités et tâches impliquées.

Résultats: Le cours d'études supérieures de deux crédits (90 heures de travail) comprend six modules dont un module d'intégration. Les modules débutent par des études de cas variées: loi sur le tabac (paquet neutre), sites d'injection supervisée, logement, réseaux intégrés de services pour personnes âgées en perte d'autonomie, programme de prévention pour les futures mères en milieux défavorisés, port obligatoire du casque de vélo. Les modules 1, 3, 4 et 5 visent l'apprentissage des différentes étapes du développement des politiques publiques : émergence, formulation et adoption, mise en œuvre et évaluation. Le module 2 porte sur l'importance des valeurs et des idéologies dans les politiques publiques. Le module d'intégration permet aux étudiants d'appliquer les connaissances acquises et aborde le rôle des experts dans les politiques publiques et les considérations éthiques. Conclusion : Le cours est intégré aux programmes d'études supérieures des universités participantes et permet à des étudiants de suivre à distance une formation innovante.

Mots-clés: Politique publique; Enseignement; Approche par compétences; Enseignement à distance; Apprentissage en ligne; Santé publique ; Programme d'études.

\section{$\lceil\rightarrow$ Abstract}

Objective: To present the process and challenges of developing an online competency-based course on public health policy using a collaborative international approach.

Methods: Five public health experts, supported by an expert in educational technology, adopted a rigorous approach to the development of the course: a needs analysis, identification of objectives and competencies, development of a pedagogical scenario for each module and target, choice of teaching methods and learning activities, material to be identified or developed, and the responsibilities and tasks involved.

Results: The 2-credit (90-hour) graduate course consists of six modules including an integration module. The modules start with a variety of case studies: tobacco law (neutral packaging), supervised injection sites, housing, integrated services for the frail elderly, a prevention programme for mothers from disadvantaged backgrounds, and the obligatory use of bicycle helmets. In modules 1, 3, 4 and 5, students learn about different stages of the public policy development process: emergence, formulation and adoption, implementation and evaluation. Module 2 focuses on the importance of values and ideologies in public policy. The integration module allows the students to apply the knowledge learned and addresses the role of experts in public policy and ethical considerations.

Conclusion: The course has been integrated into the graduate programmes of the participating universities and allows students to follow, at a distance, an innovative training programme.

Keywords: Public policy; Education; Competency-based approach; Distance education; e-learning; public health; Curriculum.

\footnotetext{
${ }^{1}$ Professeur, Département de gestion, d'évaluation et de politique en santé de l'École de santé publique de l'Université de Montréal - 7101 avenue du Parc, bureau 3014-6 - Montréal, H3N 1X9 - Canada.

${ }^{2}$ Professeur, Centre de recherche Politique et systèmes de santé - santé internationale de l'École de santé publique de l'Université libre de Bruxelles.

${ }^{3}$ Professeur, Faculté de médecine de l'Université Nice Sophia Antipolis et chef du département de Santé Publique au CHU de Nice.

${ }^{4}$ Professeur agrégé, Département de médecine sociale et préventive de l'École de santé publique de l’Université de Montréal.

${ }^{5}$ Experte en technologie éducative à l'École de santé publique de l'Université de Montréal.

${ }^{6}$ Professeure associée, Département de médecine sociale et préventive de l'École de santé publique de l’Université de Montréal.
} 


\section{Introduction}

Le développement des compétences est une condition essentielle à l'évolution des acteurs de santé publique. Les environnements de travail actuels exigent de ces acteurs une adaptation des connaissances mais également un changement de pratique professionnelle pour assumer efficacement leur mandat. C'est ainsi que les universités se voient dans l'obligation d'actualiser leurs programmes et de recourir à de nouvelles méthodes pédagogiques pour mieux répondre aux besoins du milieu professionnel et faire face à l'évolution des technologies numériques. Toutefois, ces institutions n'ont pas toujours les ressources nécessaires pour relever ces défis ; c'est pourquoi la collaboration entre universités se révèle une solution intéressante grâce à la mise en commun de l'expertise et des ressources.

C'est dans cette perspective que l'École de santé publique de l'Université de Montréal (ESPUM), l'École de santé publique de l'Université Libre de Bruxelles etle Département de santé publique de la Faculté de médecine de l'Université Nice Sophia Antipolis (UNS) ont entrepris une collaboration visant à enrichir leur programme respectif, notamment en développant en commun le cours en ligne politiques publiques en santé. Le cours a été conçu selon l'approche par compétences dans une perspective constructiviste. Ainsi, ce projet commun permet, grâce à une vision partagée des collaborateurs et de leur volonté de changement, de développer efficacement l'apprentissage en ligne dans les programmes universitaires de santé publique et d'intégrer des pratiques innovantes en pédagogie universitaire ayant une répercussion sur la qualité des formations et sur l'apprentissage.

Ce cours en ligne vise les politiques publiques (en anglais «policy») et non la politique (la politique partisane ou «politics ») ou le politique (la science politique ou « political science »). Par politique publique, on entend « tout ce qu'un gouvernement choisit de faire ou de ne pas faire » [1] Cela englobe les lois, règlements, programmes ou décisions budgétaires et ce, aux plans national, régional ou local.

L'objectif de cet article est de présenter le processus et les défis du développement d'un cours en ligne sur les politiques publiques en santé, conçu en collaboration internationale selon une approche par compétences.

\section{Méthodes}

Le cours en ligne Politiques Publiques a été développé par une équipe composée de cinq experts de santé publique accompagnés d'un expert en technologie éducative. Notons l'expertise spécifique en éthique de l'un d'entre eux et l'expérience dans le monde de la politique d'un autre membre ayant exercé les fonctions de Ministre de la santé. Tout au long du projet, un des experts a joué le rôle de chargé de projet. Deux étudiants de maîtrise ont contribué au développement d'activités d'apprentissage.

En un premier temps, une charte a été élaborée afin de préciser un ensemble de modalités importantes telles que les rôles et responsabilités de chacun des membres, les droits d'utilisation du cours dans chaque université, la diffusion du cours et sa pérennité. La collaboration s'est exprimée de la façon suivante. Nous avons profité de l'expertise particulière et de la complémentarité de chacun des membres du groupe. Celui qui tenait l'expertise dans une thématique précise proposait un ensemble d'éléments à intégrer dans le module. Les membres du groupe validaient, enrichissaient et discutaient des différences et ressemblances entre les pays afin d'arriver à un résultat commun pertinent. Le matériel pédagogique proposé présentait des exemples issus des réalités des trois pays dans le souci d'une complémentarité et non d'une redondance.

Le cours a été développé selon le processus de design pédagogique suivant: analyse, conception, développement, implantation et évaluation. L'analyse de besoins a permis de mettre en exergue le besoin de formation en politique publique et la pertinence de développer ce cours en ligne. Un tel besoin émerge clairement des compétences exigées par le Council on Education for Public Health (CEPH) [2] : 1. Discuter des multiples dimensions du processus d'élaboration de politiques, incluant la dimension éthique et les données probantes ; 2 . Proposer des stratégies permettant d'identifier les intervenants et de bâtir des coalitions et des partenariats pouvant influencer les résultats en matière de santé publique ; 3 . Plaider en faveur de politiques et de programmes politiques, sociaux et économiques qui amélioreront la santé de diverses populations; 4. Évaluer l'impact des politiques sur la santé publique et sur l'équité en santé. Même si les programmes des partenaires européens ne sont pas soumis à l'agrément du CEPH, l'analyse de leurs programmes de formation montrait aussi un besoin d'atteindre ces compétences. 
La phase de conception consistait à concevoir le scénario pédagogique qui définit les cibles d'apprentissage et décrit toutes les activités et les ressources requises pour réaliser ces activités et les productions attendues des étudiants. Il a été convenu de construire chacun des modules à partir d'une étude de cas. Le choix de méthodes pédagogiques variées avait pour but d'atteindre des personnes ayant des styles d'apprentissage différents et de favoriser l'interaction entre pairs et avec le professeur : entrevues, témoignages, matériel audio et vidéo, présentations PowerPoint narrées, forum de discussion, lectures de texte, simulations, jeux de rôle, autoévaluation, exercices d'évaluation formative et sommative. Le cours est divisé en modules dont certains peuvent être livrés dans d'autres cadres que le présent cours. L'élaboration du scénario pédagogique, qui est une étape fondamentale et itérative par ses nombreux allers et retours, a engendré au sein de l'équipe des interactions d'une grande richesse. Le matériel de formation a été développé ou choisi en tenant compte de la réalité de chacune des institutions et de chaque pays. Les vidéo et capsules audio ont été développées par l'équipe ou puisés sur des sites internet publics libres de droits. Tout au long du processus et particulièrement à cette étape, l'équipe a procédé à de nombreuses validations afin d'assurer la qualité et la pérennité de la formation. Enfin, pour la mise en ligne du matériel dans l'environnement numérique d'apprentissage, nous nous sommes assurés de la clarté des consignes pour chaque module, de la convivialité, de la facilité de la navigation, du bon fonctionnement des liens, des outils de collaboration, des tests, etc.

La mise à l'essai a été réalisée à la session d'hiver 2017 (SPU 6001) à l'Université de Montréal dans le cadre du programme de Maîtrise en santé publique. Cinq étudiantes l'ont suivi et un processus continu de monitorage a permis d'évaluer la pertinence et la qualité des outils pédagogiques.

\section{Résultats}

Le cours d'études supérieures est structuré en six modules dont un module d'intégration (tableau I). Chaque module est construit à partir d'une étude de cas. Ces cas représentent une variété de situations professionnelles d'actualité en santé publique : promotion de saines habitudes de vie (loi sur le tabac), action sur les déterminants sociaux de la santé (logement), prévention des traumatismes (port du casque de vélo), prévention chez des populations vulnérables (sites d'injection supervisée ; grossesse chez de futures mères en milieux défavorisées) et organisation des services de santé (réseau intégré de services aux personnes âgées en perte d'autonomie).

Le cours de deux crédits ( 90 heures de travail) respecte le modèle séquentiel du développement des politiques publiques proposé par Anderson [3] : mise à l'ordre du jour (ou émergence), formulation, adoption, mise en œuvre et évaluation. Le module 1 expose les notions de base en politiques publiques à partir du modèle de Kingdon [4] qui définit la fenêtre d'opportunité et les trois courants nécessaires à l'émergence des politiques publiques : problème, solution, contexte. On aborde ensuite les différents acteurs, définis selon la typologie de Lemieux [5] et la théorie des coalitions plaidantes de Sabatier et Jenkins-Smith [6]. Un aperçu des différentes stratégies, tactiques et types de preuve est aussi présenté. L'étude de cas sur le paquet neutre proposé par plusieurs pays pour renforcer la lutte antitabac permet aux étudiants d'intégrer ces notions théoriques.

Le module 2 concerne l'importance des valeurs et des idéologies politiques dans l'élaboration des politiques publiques. En l'absence d'un outil adéquat, un questionnaire d'auto-évaluation a été créé permettant aux étudiants de connaître leurs valeurs, de se situer sur un diagramme «radar» et de se comparer aux valeurs des principales idéologies politiques. Les valeurs retenues ont été sélectionnées à partir du cadre de Schwartz [7] et s'articulent selon trois axes : bienveillance $v s$ pouvoir (individualisme) ; tolérance au risque vs sécurité ; créativité vs conformité aux traditions. Les étudiants appliquent ce cadre en analysant un débat politique sur les sites d'injection supervisée. La préparation d'un mémoire en équipe sur le sujet à partir du point de vue de l'idéologie sociale-démocrate, conservatrice ou libérale et la participation à une commission parlementaire fictive où les étudiants fournissent un exposé écrit en adoptant différents rôles (ministre social-démocrate, critique conservateur ou libéral, députés de chacun des partis et leaders de chacun des trois partis) favorisent l'intégration de la matière.

Les ressources du module 3 soutiennent l'étudiant dans l'élaboration d'une politique du logement pour une municipalité de son choix en utilisant le cadre de l'OMS [8]. Le module 4 traite des théories de mise en œuvre des politiques publiques en les appliquant à l'implantation des réseaux intégrés de services pour personnes âgées en perte d'autonomie en France (MAIA) [9] et au Québec (PRISMA) [10]. Le module 5 aborde l'évaluation des politiques publiques à partir du programme « Naître égaux grandir en santé » qui vise à améliorer les habitudes de vie 


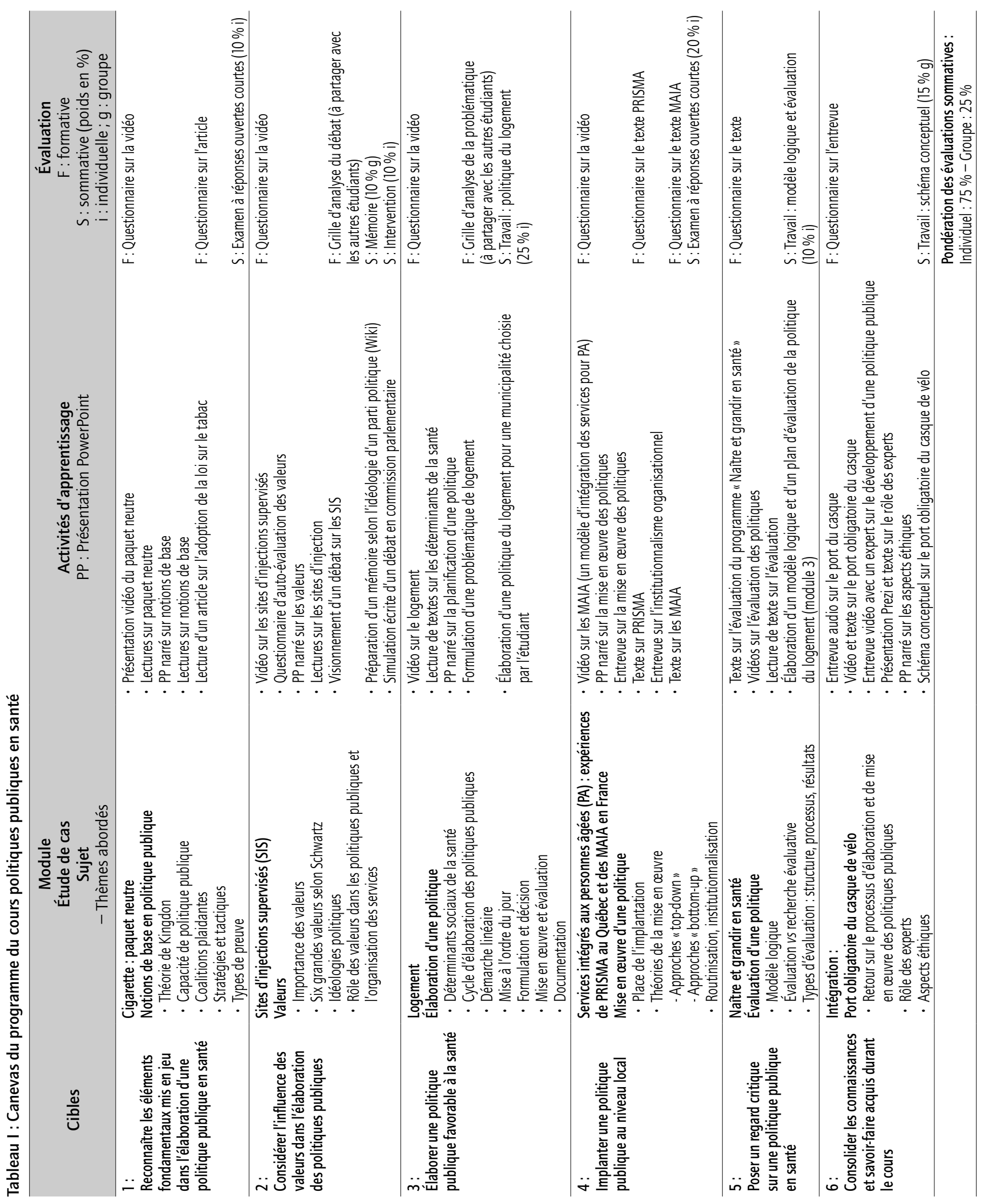


chez les futures mères en milieux défavorisés au Québec [11]. Les étudiants élaborent un modèle logique [12] et un cadre d'évaluation [13] pour la politique du logement qu'ils ont produite au module 3 .

Enfin, dans le module d'intégration les étudiants appliquent les notions acquises en relation avec la problématique internationale du port obligatoire du casque de vélo en schématisant leur conception d'une telle politique. Le rôle des experts dans l'élaboration des politiques publiques [14] et les considérations éthiques [15] y sont aussi abordés.

Dans le but de répondre aux besoins des étudiants, ces derniers peuvent s'exprimer dans un forum de questions et accéder à une section «pour en savoir davantage » qui présente des références récentes sur la problématique à l'étude et les notions traitées dans chacun des modules.

Ce cours en ligne nécessite l'implication d'un tuteur qui accompagne les apprenants tout au long de leur parcours. Le tuteur est un professionnel confirmé dans sa compétence et qui intervient à la demande (via le forum, les wikis, etc.) et lors des moments d'évaluations formatives. Il sera aussi l'évaluateur de chaque module (évaluation sommative).

Le cours utilise la plateforme Moodle qui est adaptée à ce type de travail collaboratif et qui a un potentiel pédagogique, souvent sous-exploité. Cette plateforme correspond parfaitement aux besoins pédagogiques actuels et au nécessaire tutorat pour accompagner les étudiants.

La mise à l'essai du cours auprès de cinq étudiantes a permis de révéler les éléments suivants :

- Globalement, les étudiantes ont été très satisfaites du cours. Les objectifs étaient clairs, le matériel pédagogique pertinent et la navigation facile. Les évaluations formatives et sommatives étaient appropriées.

- Des suggestions ont été faites pour ajouter un exercice formatif au module 1 , augmenter l'espace de rédaction des documents pour la commission parlementaire (module 2) et préciser certains critères d'évaluation des travaux.

- Les étudiantes ont toutes bien réussi $(\mathrm{A}+)$ mais une des étudiantes a abandonné le cours à mi-parcours pour des raisons personnelles.

\section{Discussion}

Le présent article montre comment trois universités francophones, européennes et québécoise ont collaboré afin de concevoir un cours en ligne selon une approche par compétence et sur un sujet d'intérêt commun : les politiques publiques en santé. L'enjeu était de taille car le cours en ligne devait répondre à la fois aux besoins particuliers de chacune des universités en tenant compte de leur réalité et dans le même temps lui donner une dimension assez générale pour retenir l'attention d'un auditoire plus large. Le défi consistait également à fonctionner de façon efficace malgré la distance. Le partage équitable d'expertise et de savoir-faire a également fait l'objet d'une attention constante.

Les principaux concepts mis en jeu lors de ce projet ont fait l'objet d'une clarification entre les membres afin de constituer notre cadre conceptuel et de se positionner tout au long de ce travail. Principalement, un processus de design pédagogique rigoureux (analyse de besoins, développement, mise en œuvre, évaluation) [16] ; la collaboration [17] entre les membres inspirée également du co-développement [18] ; l'approche par compétences dans laquelle la compétence est vue comme un savoir-agir complexe qui prend appui sur un ensemble de ressources [19-21] et le e-learning qui est vu comme l'utilisation des nouvelles technologies multimédias et de l'Internet pour améliorer la qualité de l'apprentissage en facilitant l'accès à des ressources et à des services, ainsi que les échanges et la collaboration à distance [22]. Il faut dire que le projet s'inscrivait dans une dynamique de progression incontestable de l'apprentissage en ligne dans les universités [23]. Certes, l'accroissement incessant des connaissances, le changement sociétal et souvent le sous-financement, poussent de nombreuses universités à explorer d'autres formes d'acquisition de connaissances et de développement des compétences [24, 25].

L'implication des membres tout au long du projet a été remarquable dans le mesure où la participation assidue aux réunions de travail virtuelles ou en présentiel était intense tant sur le plan des échanges que de la production. Le cours de deux crédits Politiques publiques en santé est constitué de six modules, ce qui représente pour l'étudiant 90 heures de travail. Il y trouve diverses activités d'apprentissage, du matériel varié et la possibilité d'interagir avec ses pairs et son tuteur. L'évaluation de l'expérimentation initiale du cours a permis de procéder aux ajustements nécessaires. Il est maintenant disponible dans d'autres programmes de l'École de santé publique de l'Université de Montréal (Maîtrise en administration de la santé, Doctorat en santé publique). Il est intégré aux programmes de Master en santé publique de l'Université de Nice Sophia Antipolis qui est disponible également sous la forme d'un diplôme universitaire. Il fait aussi partie du Master en santé publique et du certificat universitaire en formation continue de l'Université libre de Bruxelles. 
En plus de la clientèle inscrite aux programmes réguliers de santé publique, les universités cherchent à rejoindre également les professionnels de santé publique en exercice. Ces derniers cumulent les caractéristiques de la clientèle des cours en ligne soit travail, famille, vie sociale, peu de temps pour les déplacements, autonomie et flexibilité [26].

De façon générale nous n'avons pas rencontré d'obstacles majeurs. Les quelques discussions autour d'ajustements nécessaires étaient plutôt considérés comme un apprentissage collectif. En premier lieu, il s'agissait de co-construire avec des cultures universitaires différentes dotées de structures et d'une organisation pas toujours convergentes. Cependant, sur plusieurs aspects, les trois universités étaient guidées par les mêmes valeurs d'équité, d'ouverture et de partage des savoirs.

Aussi, malgré une organisation très rigoureuse : coordination efficace, rencontres virtuelles fréquentes, partage de documents à travers la plateforme et validations systématiques, une troisième rencontre non prévue, en face-à-face, a été organisée car nécessaire pour finaliser le cours. Il faut noter également quelques difficultés à respecter les échéanciers étant donné la charge de travail et les priorités qui ont surgi en cours de projet. Malgré tout, le projet s'est terminé dans les délais requis, ce qui est assez intéressant car les études montrent bien la difficulté à maintenir le rythme de production dans de telles dynamiques [27]. Mentionnons le rôle crucial joué par le soutien technopédagogique en appui aux professeurs pour la réalisation des outils d'apprentissage et la mise en ligne sur les plateformes respectives des trois universités.

Au-delà des difficultés rencontrées, les retombées positives de ce projet collaboratif sont certaines. Compte tenu de la mise en commun des expertises et des critères établis unanimement, la qualité du travail produit assure la crédibilité de la formation en ligne et permet un meilleur apprentissage. La flexibilité des formations en ligne ainsi que son potentiel d'adaptation à diverses clientèles et réalités du milieu favorisent l'équité de l'accès à la formation, notamment dans les pays en voie de développement.

En effet, dans le cadre du programme « Skill enhancement for Public Health » de l'Agence de santé publique du Canada, les résultats d'une enquête menée auprès de leaders professionnels et académiques de santé publique de trois pays de l'Afrique de l'Ouest ont montré que la qualité de l'infrastructure technologique et la connexion Internet étaient jugées suffisantes pour suivre les modules en ligne de ce programme [28].

Par ailleurs, le succès de ce projet pourra favoriser l'émergence d'autres collaborations et ainsi, contribuer à la promotion de la collaboration entre les universités. Cette collaboration permet aussi d'accroître l'usage du français dans un univers numérique de la formation en ligne largement dominé par des productions en anglais. Enfin, ce projet contribuera fortement à l'internationalisation des programmes et des universités. D'une part, les contenus québécois, belges et français exposeront les étudiants d'autres pays à la francophonie. D'autre part, ces cours permettront d'offrir des formations à des étudiants internationaux en réduisant leur temps de présence à l'étranger, ce qui constitue bien souvent une contrainte majeure.

\section{Aucun conflit d'intérêt déclaré}

\section{Remerciements}

La réalisation de ce cours a été rendue possible grâce au soutien et à la contribution financière du Fonds d'impulsion du G3, un partenariat entre les universités de Montréal, Genève et libre de Bruxelles. Il a aussi bénéficié d'une subvention du Conseil franco-québécois de coopération universitaire. Nous tenons à remercier les étudiants Mathieu Seppey (Maîtrise en santé publique de l'Université de Montréal) et Dominique Desautels (Maîtrise en politique appliquée de l'Université de Sherbrooke) de leur contribution.

\section{Références}

1. Dye TR. Understanding Public Policy. Englewood Cliffs (NJ): PrenticeHall, 1972.

2. Council on Education for Public Health. Criteria for schools and program, 2016;17-18. En ligne https://ceph.org/assets/2016. Criteria.pdf, visité le 25 mai 2017.

3. Anderson JE. Public Policy Making; New York: Holt, Rinehart and Wilson, 1984.

4. Kingdon JW. Agendas, Alternatives and Public Policies. New York: Harper Collins; 1995

5. Lemieux V. L'étude des politiques publiques. Québec : Les Presses de I'Université Laval ; 2009.

6. Sabatier PA, Jenkins-Smith HC. The Advocacy Coalition Framework: An Assessment. In: Sabatier PA, editor. Theories of the Policy Process; Boulder (Colorado): WestviewPress, 1999.

7. Schwartz SH. Les valeurs de base de la personne : théorie, mesures et applications. Revue française de sociologie. 2006;47(4): 929-68.

8. Barton $\mathrm{H}$, Tsourou C. Urbanisme et santé : Un guide de l'OMS pour un urbanisme centré sur les habitants. Genève : OMS, 2000.

9. Caisse Nationale de Solidarité pour l'Autonomie. Méthode d'action pour l'intégration des services d'aide et de soin dans le champ de l'autonomie. Paris : Les cahiers pédagogiques de la CNSA, 2014.

10. Hébert R, Raîche M, Dubois MF, Dubuc N, Gueye NR, Blanchette D et al. Les impacts du réseau intégré de services PRISMA. Gérontologie et Société. 2008;124:15-48.

11. Brodeur JM, Boyer G, Séguin L, Perreault M, Colin C, Théoret B et al. Le programme "Naître égaux - Grandir en santé " : Étude des effets 
sur la santé des mères et des nouveaux-nés. Santé, société et solidarité. 2004;3(1):119-27.

12. Morestin F, Castonguay J. Construire le modèle logique d'une politique favorable à la santé : pourquoi et comment? Montréal : Centre de collaboration nationale sur les politiques publiques et la santé, 2013.

13. Champagne F, Contandriopoulos AP, Brousselle A, Hartz Z, Denis JL. L'évaluation dans le domaine de la santé : concepts et méthodes. In : Brousselle A, Champagne F, Contandriopoulos AP, Hartz Z (editor). L'évaluation : concepts et méthodes. Montréal : Les Presses de l'Université de Montréal, 2011.

14. Morestin F. Partage de connaissances et politiques publiques : une représentation des processus d'influence. Montréal : Centre de collaboration nationale sur les politiques publiques et la santé, 2015.

15. Filiatrault F, Désy M. Référentiel de valeurs pour soutenir l'analyse éthique des actions en santé publique. Montréal : Institut national de santé publique du Québec, 2015.

16. Basque J. L'élaboration du scénario pédagogique. Texte tiré du cours : EDU 1030 Design pédagogique en formation des adultes offert en ligne. Montréal : Télé-université ; 2007. visité le 5 mai 2017.

17. Piquet $A$. Guide pratique du travail collaboratif : théories, méthodes et outils au service de la collaboration, Service "Internet et Expression Multimédia ", Ville de Brest, 2009. En ligne, http:// www.a-brest.net/IMG/pdf/Guide_pratique_du_travail_collaboratif.pdf, visité le 25 mai 2017.

18. Payette A. Le codéveloppement : une approche graduée. Interaction. 2000:4(2). En ligne, consulté le 5 mai 2017. https://www. usherbrooke.ca/psychologie/fileadmin/sites/psychologie/ espaceetudiant/Revue_Interactions/Volume_4_no_2/V4N2_ PAYETTE_Adrien_p39-60.pdf). https://www.usherbrooke.ca/ psychologie/recherche/publications/volume-4-no-2, visité le 25 mai 2017.
19. Tardif J. L'évaluation des compétences. Documenter le parcours de développement. Montréal : Chenelière Éducation, 2006.

20. Le Boterf G. Ingénierie et évaluation des compétences. Paris: Éditions d'Organisation, 2002.

21. Joannert P. Compétences et socioconstructivisme: un cadre théorique. Perspective en éducation et formation. Bruxelles: De boeck, 2009.

22. Commission of the European communities. The use of ICT to support innovation and lifelong learning for all - A report on progress. Brussels: Commission of the European communities, 2008. [En ligne]. Consulté le 30 mai 2017. http://www.europarl.europa.eu/ registre/docs_autres_institutions/commission_europeenne/ sec/2008/2629/COM_SEC(2008)2629_EN.pdf

23. Conseil supérieur de l'éducation. La formation à distance dans les universités québécoises : un potentiel à optimiser. Avis au Ministère de l'Éducation de l'Enseignement supérieur et de la Recherche, Québec, 2015.

24. Bertrand L. Renouveler l'université. Pour un rapport au savoir adapté au xxl ${ }^{\mathrm{e}}$ siècle. Québec : Les Presses de l'Université Laval, 2010.

Bates T. Teaching in a Digital Age: Guidelines for designing teaching and learning for a digital age. Vancouver: Tony Bates Associates Ltd, 2015. Consulté le 30 mai 2017: https://opentextbc.ca/ teachinginadigitalage/

25. Androni M. E-learning as part of open distance learning. Conference proceedings of "eLearning and Software for Education" (eLSE) issue. 2012;2:2732, on: www.ceeol.com

26. Farley C, Laflamme A, Raynault MF. Élaboration et expérimentation d'un microprogramme de $2^{\mathrm{e}}$ cycle en ligne. Revue Pédagogie médicale. 2009;10:S55-C062.

27. Farley C. Compétences en santé publique - La formation continue en ligne : un outil pertinent pour l'Afrique de l'Ouest. Communication orale présentée lors des $1^{\text {res }}$ Journées internationales de santé publique, Dakar, du 16 au 18 avril 2009. 